\title{
Principales resultados de la estadística sobre mortalidad en México, 1998*
}

$\mathrm{L}$ a Décima Revisión de la Clasificación Internacional de Enfermedades y Problemas Relacionados con la Salud (CIE-10) se estableció en México, en 1998. Esta nueva revisión introduce cambios sustancialmente importantes, entre éstos, destacan: a) el aumento del número de códigos; b) la modificación en la denominación de enfermedades y la reorganización de las mismas dentro de los capítulos, y c) adecuación de algunos procedimientos en la codificación y selección de las causas de defunción, lo que repercute, necesariamente, en la estadística que se presenta.

En cuanto a los cambios que deben ser tomados en cuenta para analizar el comportamiento de la mortalidad, sobresalen: a) la ampliación de 19 a 21 del número de capítulos; b) la creación, al final de algunos de los capítulos, de categorías para trastornos que son consecuencia de procedimientos médicos y, por ende, ya no se suman en el capítulo de causas externas, y c) la localización dentro del capítulo de enfermedades infecciosas y parasitarias de enfermedades como el SIDA que en la clasificación anterior se ubicaban dentro del capítulo de enfermedades endocrinas, de la nu- trición, del metabolismo y trastornos de la inmunidad.

En cuanto a los procedimientos para la codificación los cambios relevantes son: a) que ciertos tumores malignos e infecciones pueden ser aceptados como consecuencia del SIDA, en cuyo caso se selecciona a esta enfermedad como causa básica y no al tumor; b) que las neumonías y bronconeumonías pueden ser aceptadas como complicaciones terminales de enfermedades graves como tumores malignos, desnutrición, enfermedades paralizantes y traumatismos graves y, con ello, disminuye la posibilidad de seleccionar a la neumonía y bronconeumonía como causa básica por lo que disminuirá su número por efectos del cambio en la codificación.

Como en años anteriores se presentan seis cuadros con información relevante sobre la mortalidad para 1998. En el cuadro I la mortalidad general por sexo, no se aprecian grandes variaciones en cuanto al número y las tasas respecto al año anterior. En el segundo, que se refiere a la distribución de las defunciones por grupo de edad, se aprecia la continua reducción del número de defunciones en los menores de 24 años mientras que aumenta el peso relativo de los grupos de 65 años y más. El cuadro III permite comparar con años anteriores el proceso de transición epidemiológica al observar el creciente aumento de las enfermedades transmisibles y la disminución de las no transmisibles, con cambio mínimo en la proporción de las lesiones. Los cuadros IV y VI presentan la distribución de las veinte principales causas de defunción, su tasa y su peso relativo con carácter nacional y el comportamiento de algunas enfermedades infecciosas por entidad federativa, respectivamente. El cuadro V presenta para cada entidad la edad promedio a la muerte y los años de vida potencial perdidos debido a las defunciones que ocurren en edades previas respecto a una esperanza de vida promedio de 70 años.

La Dirección General de Estadística e Informática pone a disposición de los lectores el anuario correspondiente para ampliar la información con relación a otros aspectos importantes de la mortalidad, mismos que se pueden consultar en el Centro de Referencia de Información de Estadística en Salud (CRIES), ubicado en Homero 213, primer piso, colonia Chapultepec Morales, 11570 México, D.F. 


\section{Cuadro I \\ Mortalidad general por SeXo, SEgún entidad federativa de ResidenCia habitual. MÉXICO, 1998}

\begin{tabular}{|c|c|c|c|c|c|c|c|c|}
\hline & \multicolumn{2}{|c|}{ Total } & \multicolumn{2}{|c|}{ Hombres } & \multicolumn{2}{|c|}{ Mujeres } & \multirow[b]{2}{*}{$N E$} & \multirow[b]{2}{*}{ ISM } \\
\hline Entidad federativa & Defunciones & Tasa & Defunciones & Tasa & Defunciones & Tasa & & \\
\hline Estados Unidos Mexicanos & 444665 & 4.6 & 249030 & 5.2 & 195460 & 4.0 & 175 & 127.4 \\
\hline Aguascalientes & 3783 & 4.0 & 2064 & 4.5 & 1719 & 3.6 & 0 & 120.1 \\
\hline Baja California & 10910 & 4.8 & 6825 & 6.0 & 4079 & 3.6 & 6 & 167.3 \\
\hline Baja California Sur & 1553 & 3.9 & 928 & 4.6 & 625 & 3.3 & 0 & 148.5 \\
\hline Campeche & 2709 & 4.0 & 1632 & 4.7 & 1077 & 3.2 & 0 & 151.5 \\
\hline Coahuila & 10202 & 4.4 & 5615 & 4.8 & 4585 & 4.0 & 2 & 122.5 \\
\hline Colima & 2370 & 4.5 & 1317 & 5.1 & 1051 & 4.0 & 2 & 125.3 \\
\hline Chiapas & 16413 & 4.2 & 9466 & 4.8 & 6940 & 3.5 & 7 & 136.4 \\
\hline Chihuahua & 15386 & 5.2 & 9048 & 6.1 & 6331 & 4.3 & 7 & 142.9 \\
\hline Distrito Federal & 46773 & 5.4 & 24309 & 5.7 & 22450 & 5.0 & 14 & 108.3 \\
\hline Durango & 6106 & 4.1 & 3618 & 4.8 & 2485 & 3.3 & 3 & 145.6 \\
\hline Guanajuato & 21759 & 4.6 & 11794 & 5.1 & 9960 & 4.1 & 5 & 118.4 \\
\hline Guerrero & 10972 & 3.6 & 6443 & 4.2 & 4522 & 2.9 & 7 & 142.5 \\
\hline Hidalgo & 10136 & 4.5 & 5669 & 5.1 & 4466 & 4.0 & 1 & 126.9 \\
\hline Jalisco & 30682 & 4.8 & 16620 & 5.3 & 14050 & 4.4 & 12 & 118.3 \\
\hline México & 52095 & 4.1 & 29044 & 4.7 & 23026 & 3.6 & 25 & 126.1 \\
\hline Michoacán & 18466 & 4.5 & 10327 & 5.1 & 8131 & 3.9 & 8 & 127.0 \\
\hline Morelos & 6848 & 4.5 & 3860 & 5.1 & 2983 & 3.8 & 5 & 129.4 \\
\hline $\mathrm{N}$ ayarit & 4119 & 4.4 & 2392 & 5.1 & 1724 & 3.7 & 3 & 138.7 \\
\hline Nuevo León & 15936 & 4.3 & 8842 & 4.7 & 7087 & 3.8 & 7 & 124.8 \\
\hline 0 axaca & 18565 & 5.3 & 10453 & 6.0 & 8111 & 4.6 & 1 & 128.9 \\
\hline Puebla & 26413 & 5.3 & 14568 & 6.0 & 11833 & 4.7 & 12 & 123.1 \\
\hline Q uerétaro & 5950 & 4.4 & 3313 & 8.4 & 2634 & 7.0 & 3 & 125.8 \\
\hline Q uintana Roo & 2083 & 2.7 & 1269 & 1.9 & 807 & 1.2 & 7 & 157.2 \\
\hline San Luis Potosí & 10501 & 4.5 & 5807 & 5.0 & 4690 & 4.0 & 4 & 123.8 \\
\hline Sinaloa & 10230 & 4.1 & 6134 & 4.9 & 4093 & 3.3 & 3 & 149.9 \\
\hline Sonora & 10491 & 4.8 & 6193 & 5.6 & 4298 & 4.0 & 0 & 144.1 \\
\hline Tabasco & 7789 & 4.2 & 4598 & 4.9 & 3189 & 3.4 & 2 & 144.2 \\
\hline Tamaulipas & 11548 & 4.3 & 6577 & 5.0 & 4964 & 3.7 & 7 & 132.5 \\
\hline Tlaxcala & 4356 & 4.6 & 2348 & 5.0 & 2007 & 4.2 & 1 & 117.0 \\
\hline Veracruz & 32424 & 4.6 & 18232 & 5.3 & 14175 & 4.0 & 17 & 128.6 \\
\hline Yucatán & 8318 & 5.1 & 4501 & 5.5 & 3816 & 4.7 & 1 & 118.0 \\
\hline Zacatecas & 6580 & 4.7 & 3703 & 5.3 & 2876 & 4.0 & 1 & 128.8 \\
\hline Extranjero & 2199 & & 1521 & & 676 & & 2 & 225.0 \\
\hline
\end{tabular}

Tasa por 1000 habitantes según sexo

Fuente: Instituto N acional de Estadística, Geografía e Informática y Dirección General de Estadística e Informática. Secretaría de Salud, México

$\mathrm{NE}=$ no especificado

ISM = índice de sobremortalidad masculina 


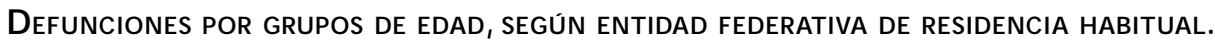 MÉXICO, 1998}

\begin{tabular}{|c|c|c|c|c|c|c|c|c|c|c|c|}
\hline Entidad federativa & Total & -1 año & $1-4$ & $5-14$ & $15-24$ & $25-34$ & $35-44$ & $45-54$ & $55-64$ & $65 y+$ & NE \\
\hline Estados Unidos Mexicanos & 444665 & 42183 & 8541 & 7882 & 19426 & 24761 & 30376 & 38923 & 55033 & 215020 & 2520 \\
\hline Aguascalientes & 3783 & 403 & 68 & 78 & 150 & 169 & 208 & 274 & 421 & 1983 & 29 \\
\hline Baja California & 10910 & 1035 & 178 & 176 & 622 & 936 & 1089 & 1057 & 1417 & 4307 & 93 \\
\hline Baja California Sur & 1553 & 124 & 27 & 19 & 87 & 91 & 115 & 137 & 205 & 719 & 29 \\
\hline Campeche & 2709 & 237 & 48 & 39 & 112 & 176 & 176 & 234 & 317 & 1332 & 38 \\
\hline Coahuila & 10202 & 570 & 88 & 131 & 397 & 439 & 630 & 933 & 1500 & 5486 & 28 \\
\hline Colima & 2370 & 173 & 37 & 33 & 77 & 105 & 161 & 224 & 273 & 1262 & 25 \\
\hline Chiapas & 16413 & 1897 & 658 & 545 & 1169 & 1288 & 1413 & 1493 & 1756 & 6005 & 189 \\
\hline Chihuahua & 15386 & 1265 & 261 & 239 & 815 & 986 & 1083 & 1346 & 2083 & 7148 & 160 \\
\hline D istrito Federal & 46773 & 3699 & 445 & 440 & 1520 & 2394 & 3069 & 4251 & 6102 & 24840 & 13 \\
\hline Durango & 6106 & 157 & 63 & 107 & 262 & 314 & 372 & 509 & 860 & 3416 & 46 \\
\hline Guanajuato & 21759 & 2824 & 474 & 423 & 815 & 956 & 1230 & 1622 & 2271 & 11002 & 142 \\
\hline Guerrero & 10972 & 737 & 294 & 274 & 693 & 901 & 962 & 1025 & 1331 & 4668 & 87 \\
\hline Hidalgo & 10136 & 983 & 187 & 201 & 426 & 523 & 726 & 981 & 1276 & 4739 & 94 \\
\hline Jalisco & 30682 & 2476 & 450 & 553 & 1180 & 1492 & 1792 & 2441 & 3462 & 16661 & 175 \\
\hline México & 52095 & 7634 & 1086 & 921 & 2614 & 3219 & 4153 & 5164 & 6509 & 20623 & 172 \\
\hline Michoacán & 18466 & 1357 & 345 & 348 & 875 & 973 & 1142 & 1400 & 2019 & 9930 & 77 \\
\hline Morelos & 6848 & 565 & 105 & 70 & 280 & 388 & 436 & 571 & 824 & 3534 & 75 \\
\hline $\mathrm{N}$ ayarit & 4119 & 162 & 64 & 73 & 170 & 197 & 210 & 336 & 523 & 2365 & 19 \\
\hline Nuevo León & 15936 & 1186 & 172 & 223 & 512 & 721 & 887 & 1380 & 2328 & 8429 & 98 \\
\hline 0 axaca & 18565 & 1705 & 676 & 442 & 833 & 1127 & 1348 & 1623 & 2171 & 8557 & 83 \\
\hline Puebla & 26413 & 3794 & 749 & 531 & 1067 & 1275 & 1752 & 2297 & 2988 & 11863 & 97 \\
\hline Q uerétaro & 5950 & 759 & 134 & 125 & 274 & 342 & 428 & 484 & 694 & 2656 & 54 \\
\hline Q uintana Roo & 2083 & 303 & 50 & 57 & 136 & 188 & 186 & 215 & 219 & 675 & 54 \\
\hline San Luis Potosí & 10501 & 1004 & 215 & 213 & 409 & 453 & 555 & 745 & 1262 & 5621 & 24 \\
\hline Sinaloa & 10230 & 312 & 129 & 151 & 524 & 609 & 698 & 863 & 1339 & 5551 & 54 \\
\hline Sonora & 10491 & 833 & 151 & 160 & 404 & 553 & 670 & 946 & 1530 & 5207 & 37 \\
\hline Tabasco & 7789 & 919 & 190 & 219 & 458 & 500 & 579 & 681 & 932 & 3255 & 56 \\
\hline Tamaulipas & 11548 & 734 & 131 & 171 & 473 & 672 & 690 & 1004 & 1685 & 5937 & 51 \\
\hline Tlaxcala & 4356 & 675 & 90 & 98 & 167 & 175 & 241 & 342 & 486 & 2058 & 24 \\
\hline Veracruz & 32424 & 2512 & 701 & 570 & 1210 & 1773 & 2302 & 3015 & 4348 & 15771 & 222 \\
\hline Yucatán & 8318 & 651 & 133 & 103 & 201 & 295 & 433 & 566 & 918 & 4886 & 132 \\
\hline Zacatecas & 6580 & 468 & 122 & 110 & 266 & 245 & 335 & 491 & 682 & 3822 & 39 \\
\hline Extranjero & 2199 & 30 & 20 & 39 & 228 & 286 & 305 & 273 & 302 & 712 & 4 \\
\hline
\end{tabular}

Fuente: Instituto N acional de Estadística, Geografía e Informática y Dirección General de Estadística e Informática. Secretaría de Salud, México

$N E=$ no especificado 


\section{Cuadro III \\ Defunciones POR enfermedAdeS TRANSMISIBLES, NO TRANSMISIBLES Y LESIONES. MÉxıco, 1998}

Entidad federativa

Estados Unidos Mexicanos

Aguascalientes

Baja California

Baja California Sur

Campeche

Coahuila

Colima

Chiapas

Chihuahua

Distrito Federal

Durango

Guanajuato

Guerrero

Hidalgo

Jalisco

México

Michoacán

Morelos

$\mathrm{N}$ ayarit

Nuevo León

0 axaca

Puebla

Q uerétaro

Q uintana Roo

San Luis Potosí

Sinaloa

Sonora

Tabasco

Tamaulipas

Tlaxcala

Veracruz

Yucatán

Zacatecas

Extranjero
Total

444665

Subtotal*

437100

3783

10910

1553

2709

10202

2370

16413

15386

46773

6106

21759

10972

10136

30682

52095

18466

6848

4119

15936

18565

26413

5950

2083

10501

10230

10491

7789

11548

4356

32424

8318

6580

2199
3756

10784

1539

2597

10098

2356

15621

15101

46500

5926

21526

10669

9961

30428

51855

18140

6799

4059

15700

17765

25417

5858

2054

10209

10054

10386

7685

11438

4260

31968

8064

6431

2096
Enfermedades transmisibles ${ }^{\ddagger}$ Defunciones

42290

9.7

$392 \quad 10.4$

$1126 \quad 10.4$

$150 \quad 9.7$

$254 \quad 9.8$

$719 \quad 7.1$

$196 \quad 8.3$

$2486 \quad 15.9$

1222

3795

263

2341

1128

128

878

2510

5925

1353
672

$672 \quad 9.9$

$281 \quad 6.9$

$1254 \quad 8.0$

2125

2125

3143

$676 \quad 11.5$

$314 \quad 15.3$

$1031 \quad 10.1$

$586 \quad 5$

$806 \quad 7.8$

982

982
844

$\begin{array}{rr}982 & 12.8\end{array}$

$461 \quad 10.8$

$3093 \quad 9.7$

$781 \quad 9.7$

$\begin{array}{ll}429 & 6.7\end{array}$

74

* El subtotal no incluye los signos, síntomas y estados morbosos mal definidos (7 565). Es a partir de esta cifra que se calcula la distribución porcentual

₹ Incluye enfermedades infecciosas y parasitarias, muertes maternas y perinatales

$\S$ Se refiere a las causas externas de morbilidad y mortalidad

Fuente: Instituto N acional de Estadística, Geografía e Informática y Dirección General de Estadística e Informática. Secretaría de Salud, México 


\section{Cuadro IV \\ Principales causas de mortalidad general. \\ MÉXICO, 1998}

Número

de orden

Causa

Total

Enfermedades del corazón

444665

460.1

100.0

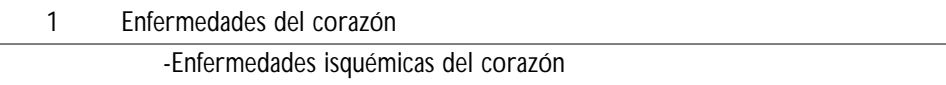

\begin{tabular}{cl}
\hline 2 & Tumores malignos \\
\hline 3 & Diabetes mellitus \\
\hline 4 & Accidentes \\
\hline & - Accidentes de tráfico de vehículos de motor \\
\hline
\end{tabular}

$100-151$ exc. 146

$68677 \quad 71.1 \quad 15.4$

$120-125$

$42873 \quad 44.4 \quad 9.6$

C00-C 97

$52670 \quad 54.5 \quad 11.8$

E10-E14

41832

V01-X $59, Y 40-Y 86$

35517

Varias ${ }^{\ddagger}$

11541

43.3

36.8

9.4

5 Enfermedades del hígado
- Enfermedad alcohólica del hígado

6 Enfermedades cerebrovasculares

K $70-K 76$

27211

K70

13619

$160-169$

25050

P00-P96,A 33

19879

P35-P37

2998

- Enfermedades infecciosas y parasitarias congénitas

P20-P21

$\begin{array}{lll}1759 & 1.8 & 0.4\end{array}$

- Crecimiento fetal lento, desnutrición fetal y trastornos relacionados con la gestación corta y el bajo peso al nacer

P05, P07

1305

J10-J18

$15026 \quad 15.6-3.4$

\begin{aligned} & \hline 8 Influenza y neumonía \\ & \hline 9 Agresiones (homicidio) \\ & \hline 10 Desnutrición y otras deficiencias nutricionales \\ & \hline 11 Enfermedades pulmonares obstructivas crónicas \\ & \hline 12 Malformaciones congénitas, deformidades y anomalías cromosómicas \\ & \hline 13 Insuficiencia renal \\ & \hline 14 Bronquitis crónica y la no especificada, enfisema y asma \\ & \hline 16 Enfermedades infecciosas intestinales \\ & \hline 17 Enfermedad por virus de la inmunodeficiencia humana \\ & \hline 18 (SIDA) \\ & \hline\end{aligned}

\begin{tabular}{ll}
\hline 19 & Lesiones autoinfligidas intencionalmente (suicidio) \\
\hline 20 & Septicemia
\end{tabular}

Paro cardíaco

0.5

Síntomas signos y hallazgos anormales clínicos y de laboratorio

no clasificados en otra parte

R00-R99

$7565 \quad 7.8$

1.7

Las demás causas

$64364 \quad 66.6$

14.5

* Tasa por 100000 habitantes. Estimada con base en las proyecciones de población del Consejo N acional de Población

₹ Comprende múltiples categorías y subcategorías

Fuente: Instituto N acional de Estadística, Geografía e Informática y Dirección General de Estadística e Informática. Secretaría de Salud, México 


\section{Cuadro V

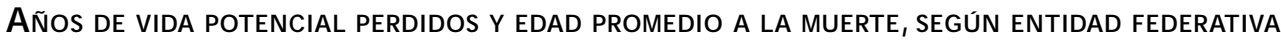 de residencia habitual. MÉXICO, 1998}

\begin{tabular}{|c|c|c|c|c|c|c|}
\hline Entidad federativa & Total & Tasa* & $\begin{array}{c}\text { Edad promedio } \\
\text { a la muerte }\end{array}$ & $\begin{array}{c}\text { Años de vida } \\
\text { potencial perdidos }\end{array}$ & $\begin{array}{l}\text { Tasa } \\
\text { AVPp }\end{array}$ & $\begin{array}{l}\% \text { respecto al } \\
\text { total de AVPP }\end{array}$ \\
\hline Estados Unidos Mexicanos & 444665 & 4.6 & 56.0 & 8231211 & 85.2 & 100.0 \\
\hline Aguascalientes & 3783 & 4.0 & 56.9 & 67786 & 72.2 & 0.8 \\
\hline Baja California & 10910 & 4.8 & 51.4 & 232798 & 102.5 & 2.8 \\
\hline Baja California Sur & 1553 & 3.9 & 56.0 & 27885 & 70.9 & 0.3 \\
\hline Campeche & 2709 & 4.0 & 56.8 & 48072 & 70.4 & 0.6 \\
\hline Coahuila & 10202 & 4.4 & 60.3 & 144399 & 62.3 & 1.8 \\
\hline Colima & 2370 & 4.5 & 59.6 & 36850 & 70.7 & 0.4 \\
\hline Chiapas & 16413 & 4.2 & 48.1 & 411385 & 104.7 & 5.0 \\
\hline Chihuahua & 15386 & 5.2 & 55.4 & 282036 & 95.3 & 3.4 \\
\hline Distrito Federal & 46773 & 5.4 & 59.3 & 728125 & 83.4 & 8.8 \\
\hline Durango & 6106 & 4.1 & 62.4 & 77655 & 51.7 & 0.9 \\
\hline Guanajuato & 21759 & 4.6 & 55.7 & 426939 & 90.3 & 5.2 \\
\hline Guerrero & 10972 & 3.6 & 53.5 & 222579 & 72.1 & 2.7 \\
\hline Hidalgo & 10136 & 4.5 & 55.6 & 190383 & 84.7 & 2.3 \\
\hline Jalisco & 30682 & 4.8 & 59.3 & 494120 & 78.0 & 6.0 \\
\hline México & 52095 & 4.1 & 50.0 & 1215797 & 96.8 & 14.8 \\
\hline Michoacán & 18466 & 4.5 & 59.1 & 305793 & 74.3 & 3.7 \\
\hline Morelos & 6848 & 4.5 & 58.3 & 113600 & 74.3 & 1.4 \\
\hline $\mathrm{N}$ ayarit & 4119 & 4.4 & 62.1 & 55023 & 58.6 & 0.7 \\
\hline Nuevo León & 15936 & 4.3 & 59.5 & 241299 & 64.4 & 2.9 \\
\hline 0 axaca & 18565 & 5.3 & 54.2 & 374065 & 106.8 & 4.5 \\
\hline Puebla & 26413 & 5.3 & 52.4 & 581788 & 117.0 & 7.1 \\
\hline Q uerétaro & 5950 & 4.4 & 53.1 & 126650 & 93.7 & 1.5 \\
\hline Q uinatana Roo & 2083 & 2.7 & 45.6 & 54409 & 70.8 & 0.7 \\
\hline San Luis Potosí & 10501 & 4.5 & 58.4 & 180629 & 77.1 & 2.2 \\
\hline Sinaloa & 10230 & 4.1 & 61.0 & 142538 & 57.6 & 1.7 \\
\hline Sonora & 10491 & 4.8 & 57.2 & 175581 & 80.4 & 2.1 \\
\hline Tabasco & 7789 & 4.2 & 51.5 & 174141 & 93.1 & 2.1 \\
\hline Tamaulipas & 11548 & 4.3 & 59.0 & 179573 & 67.6 & 2.2 \\
\hline Tlaxcala & 4356 & 4.6 & 53.5 & 93539 & 98.3 & 1.1 \\
\hline Veracruz & 32424 & 4.6 & 57.1 & 564678 & 80.7 & 6.9 \\
\hline Yuacatán & 8318 & 5.1 & 61.7 & 116925 & 71.8 & 1.4 \\
\hline Zacatecas & 6580 & 4.7 & 61.5 & 97812 & 69.3 & 1.2 \\
\hline
\end{tabular}

* Tasa por 1000 habitantes

₹ Es la suma de los años de vida potencial perdidos por cada una de las defunciones ocurridas, respecto a un límite superior de la edad de 70 años (esperanza de vida)

$\S$ Tasa de años de vida potencial perdidos por 1000 habitantes, estimada con base en las proyecciones de población de CO NAPO

Fuente: D irección General de Estadística e Informática. Secretaría de Salud, México 


\section{Cuadro VI}

\section{MORTALIDAD POR ENFERMEDADES INFECCIOSAS INTESTINALES, INFECCIONES RESPIRATORIAS AGUDAS Y SÍNDROME DE INMUNOdEFICIENCIA ADQUIRIDA, SEGÚN ENTIDAD FEDERATIVA DE RESIDENCIA HABITUAL. MÉXICO, 1998}

\begin{tabular}{|c|c|c|c|c|c|c|}
\hline \multirow[b]{2}{*}{ Entidad federativa } & \multicolumn{2}{|c|}{ Enfermedades infecciosas intestinales* } & \multicolumn{2}{|c|}{ Infecciones respiratorias agudas ${ }^{\ddagger}$} & \multicolumn{2}{|c|}{ SIDA } \\
\hline & Defunciones & Tasa & Defunciones & Tasa & Defunciones & Tasa \\
\hline Estados Unidos Mexicanos & 6668 & 6.9 & 17657 & 18.3 & 4100 & 4.2 \\
\hline Aguascalientes & 61 & 6.5 & 89 & 9.5 & 23 & 2.5 \\
\hline Baja California & 75 & 3.3 & 308 & 13.6 & 207 & 9.1 \\
\hline Baja California Sur & 14 & 3.6 & 35 & 8.9 & 19 & 4.8 \\
\hline Campeche & 29 & 4.2 & 82 & 12.0 & 26 & 3.8 \\
\hline Coahuila & 84 & 3.6 & 232 & 10.0 & 61 & 2.6 \\
\hline Colima & 21 & 4.0 & 62 & 11.9 & 28 & 5.4 \\
\hline Chiapas & 880 & 22.4 & 1056 & 26.9 & 100 & 2.5 \\
\hline Chihuahua & 134 & 4.5 & 572 & 19.3 & 93 & 3.1 \\
\hline Distrito Federal & 299 & 3.4 & 1940 & 22.2 & 773 & 8.9 \\
\hline Durango & 42 & 2.8 & 189 & 12.6 & 23 & 1.5 \\
\hline Guanajuato & 410 & 8.7 & 967 & 20.4 & 89 & 1.9 \\
\hline Guerrero & 268 & 8.7 & 311 & 10.1 & 147 & 4.8 \\
\hline Hidalgo & 101 & 4.5 & 463 & 20.6 & 39 & 1.7 \\
\hline Jalisco & 250 & 3.9 & 1045 & 16.5 & 363 & 5.7 \\
\hline México & 855 & 6.8 & 2970 & 23.7 & 480 & 3.8 \\
\hline Michoacán & 224 & 5.4 & 625 & 15.2 & 91 & 2.2 \\
\hline Morelos & 99 & 6.5 & 190 & 12.4 & 89 & 5.8 \\
\hline $\bar{N}$ ayarit & 28 & 3.0 & 106 & 11.3 & 34 & 3.6 \\
\hline Nuevo León & 102 & 2.7 & 434 & 11.6 & 149 & 4.0 \\
\hline 0 axaca & 672 & 19.2 & 910 & 26.0 & 128 & 3.7 \\
\hline Puebla & 567 & 11.4 & 1673 & 33.6 & 222 & 4.5 \\
\hline Q uerétaro & 120 & 8.9 & 199 & 14.7 & 35 & 2.6 \\
\hline Q uinatana Roo & 26 & 3.4 & 45 & 5.9 & 36 & 4.7 \\
\hline San Luis Potosí & 185 & 7.9 & 371 & 15.8 & 36 & 1.5 \\
\hline Sinaloa & 82 & 3.3 & 227 & 9.2 & 69 & 2.8 \\
\hline Sonora & 105 & 4.8 & 270 & 12.4 & 59 & 2.7 \\
\hline Tabasco & 127 & 6.8 & 274 & 14.6 & 86 & 4.6 \\
\hline Tamaulipas & 68 & 2.6 & 247 & 9.3 & 68 & 2.6 \\
\hline Tlaxcala & 70 & 7.4 & 243 & 25.5 & 26 & 2.7 \\
\hline Veracruz & 468 & 6.7 & 893 & 12.8 & 377 & 5.4 \\
\hline Yuacatán & 126 & 7.7 & 320 & 19.6 & 94 & 5.8 \\
\hline Zacatecas & 65 & 4.6 & 291 & 20.6 & 12 & 0.8 \\
\hline Extranjero & 11 & & 18 & & 18 & \\
\hline
\end{tabular}

Tasa por 100000 habitantes

* Códigos A00-A09 de la CIE-10

₹Códigos J00-J22 de la CIE-10

Fuente: Instituto N acional de Estadística, G eografía e Informática y Dirección General de Estadística e Informática. Secretaría de Salud, México 